Int. J. Odontostomat., 9(1):165-171, 2015.

\title{
Revisión Sistemática sobre los Efectos Adversos de la Fluoración del Agua
}

\author{
Systematic Review of the Adverse Effects of Water Fluoridation
}

\begin{abstract}
Carlos Zaror ${ }^{*, *} ;$ Carlos Vallejos ${ }^{* *}$; Gilda Corsini" ${ }^{* *}$ Catherine de la Puente ${ }^{\star *}$; Mónica Velásquez*; Roxana Tessada-Sepúlveda ${ }^{* * *}$; Carolina del Valle-Aranda ${ }^{* * * *}$ \& Gabriel Vega-Rodríguez ${ }^{* * *}$
\end{abstract}

ZAROR, C.; VALLEJOS, C.; CORSINI, G.; DE LA PUENTE, C.; VELÁSQUEZ, M.; TESSADA-SEPÚLVEDA, R.; DEL VALLE-ARANDA, C. \& VEGA-RODRÍGUEZ, G. Revisión sistemática sobre los efectos adversos de la fluoración del agua. Int. J. Odontostomat., 9(1):165-171, 2015.

RESUMEN: Durante las últimas décadas, una considerable atención científica ha sido puesta en la seguridad de los fluoruros, dada la amplia variedad de fuentes de ingestión a la que la población se encuentra expuesta y los riesgos a la salud de las personas que esto puede acarrear. El objetivo de esta investigación fue determinar si la fluoración del agua a concentraciones de 0,6 a 1 ppm se asocian a una mayor proporción de efectos adversos en la población general al compararlo con concentraciones subóptimas. Se realizó una revisión sistemática de la literatura en MEDLINE, EMBASE, COCHRANE, SCIELO, LILACS, CRD, BBO, PAHO y WHOLIS, limitada desde el 2002 al 2012. Se incluyeron estudios primarios y secundarios en español, inglés y portugués con al menos dos poblaciones comparadas, una con niveles óptimos de flúor en agua $(0,6-1 \mathrm{ppm})$ y otra sin fluoración del agua $(<0,3 \mathrm{ppm})$ o con niveles subóptimos $(>0,3<0,6 \mathrm{ppm})$. Dos investigadores de forma independiente realizaron evaluación de la calidad de los artículos seleccionados y que cumplieron los criterios de inclusión. La búsqueda arrojó 1024 artículos de los cuales 24 cumplieron los criterios de inclusión y 10 fueron incluidos como evidencia. Con excepción de fluorosis dental, no hay asociación entre fluoración del agua con fracturas óseas, cáncer u otro efecto adverso. A pesar de la mayor prevalencia de fluorosis en zonas fluoradas, esta fue principalmente del tipo cuestionable a leve y la proporción de fluorosis con daño estético no difiere significativamente de la presente en zonas sin fluoración del agua.

PALABRAS CLAVE: fluoruro; fluoridación, agua potable; fluorosis; efectos adversos.

\section{INTRODUCCIÓN}

Hay fuerte evidencia que la fluoración del agua reduce considerablemente la inequidad en salud oral, siendo la intervención más ampliamente implementada en salud pública para la prevención de la caries dental (McDonagh et al., 2000; Armfield, 2010). Es por esto que el Centro para el Control y la Prevención de Enfermedades de Estados Unidos (CDC) la reconoce como uno de los 10 principales logros en Salud Pública del siglo XX (CDC, 1999).

Durante las últimas décadas, una considerable atención científica ha sido puesta en la segu- ridad y efectividad de los fluoruros, dada la amplia variedad de fuentes de ingestión a la que la población se encuentra expuesta y los riesgos a la salud de las personas que esto puede acarrear (Khan et al., 2004; Levy et al., 1995, 2001).

Los beneficios de la fluoración del agua han sido acompañados de un aumento de la fluorosis dental con prevalencias que alcanzan el 32\% (Clark et al., 2006; Khan et al., 2005). Sin embargo en los últimos años se ha observado también un aumento de la prevalencia de fluorosis dental en comunidades no fluoradas (Mascarenhas \& Burt, 1998).

Departamento de Odontopediatria y Ortodoncia, Facultad de Odontología, Universidad de La Frontera, Temuco, Chile

* Centro de Excelencia CIGES, Facultad de Medicina. Universidad de La Frontera. Temuco, Chile

*** Departamento de Salud Ambiental, Subsecretaría de Salud Pública, Ministerio de Salud, Chile

**** Departamento de Salud Bucal, Subsecretaría de Salud Pública, Ministerio de Salud, Chile

El estudio fue financiado por el Ministerio de Salud de Chile, quien autoriza la publicación de los datos. 
La fluorosis dentaria es una intoxicación crónica por flúor durante el periodo de formación del esmalte dentario. Clínicamente está caracterizada por opacidades difusas en parte o todo el esmalte, que no sólo producen problemas estéticos, sino también alteraciones funcionales producto de la pérdida de estructura del esmalte en los casos más severos (Denbesten \& Li, 2011)

La fluoración del agua también ha sido asociada a otros efectos adversos como fracturas óseas, cáncer, Síndrome de Down, muerte fetal, malformaciones congénitas, mortalidad infantil, deterioro cognitivo, Alzheimer, entre otros. Sin embargo la evidencia científica no ha sido concluyente en establecer estas asociaciones (McDonagh et al.).

Si bien no hay clara evidencia de otro potencial efecto adverso (McDonagh et al.), el incremento de fluorosis dental tanto en poblaciones fluoradas como no fluoradas, ha hecho que las autoridades de salud de Chile realicen actualizaciones periódicas de la última evidencia disponible sobre el tema (Vallejos et al., 2002).

El último reporte Ministerial del año 2002, concluye que el principal efecto adverso de la fluoración de las aguas de bebida es la fluorosis dental, la cual se presenta con una prevalencia moderada, aparentemente con relación dosis-respuesta a las dosis de flúor presentes en el agua y a la asociación con exposición a otras fuentes de flúor. Además sostiene que no hay evidencia para relacionar otros efectos adversos a la fluoración del agua, considerándola una medida segura según la evidencia científica disponible (Vallejos et al.).

Con el fin de actualizar la última revisión del tema, la Subsecretaría de Salud Pública del Ministerio de Salud de Chile, encomienda la realización de este estudio, cuyo objetivo fue determinar si la fluoración del agua a concentraciones de 0,6 a 1 ppm se asocian a una mayor proporción de efectos adversos en la población general al compararlo con concentraciones subóptimas.

\section{MATERIAL Y MÉTODO}

Se realizó una revisión sistemática de la literatura en MEDLINE, EMBASE, COCHRANE, SCIELO, LILACS, CRD, BBO, PAHO y WHOLIS, limitada des- de el 2002 al 2012 sobre los efectos adversos de la fluoración de agua a concentraciones de 0,6 a 1 ppm. en población infantil, adolescente y adulta.

La búsqueda fue realizada simultáneamente por dos investigadores, en forma independiente, utilizando a la vez estrategias sensibles y específicas. Las palabras clave utilizadas fueron fluoride, fluorine, fluorene, fluoridation, topical fluoride, water, drinking water, fluorosis, bone fractures, neoplasms, cancer, adverse effects, toxicity.

Se incluyeron estudios primarios y secundarios en español, inglés y portugués con al menos dos poblaciones comparadas, una con niveles óptimos de flúor en agua $(0,6-1 \mathrm{ppm})$ y otra sin fluoración del agua $(<0,3 \mathrm{ppm})$ o con niveles subóptimos $(>0,3<0,6 \mathrm{ppm})$. Se consideró como principales medidas de resultado la prevalencia de fluorosis dental medido a través de los índices de Dean, Tylstrup-Fejerskov o TSIF, incidencia de cáncer, prevalencia de fracturas óseas o de cualquier otro efecto adverso descrito en la literatura.

Se excluyeron revisiones narrativas, reporte de casos, cartas al editor, estudios ecológicos, experimentales, in vitro y en animales.

Una vez terminada la búsqueda, una selección primaria fue realizada por dos investigadores en forma independiente, quienes revisaron los títulos y los resúmenes de los artículos resultados de la búsqueda. Los registros relevante considerados por al menos un revisor y que cumplieron los criterios de selección determinados fueron examinados.

Dos investigadores de forma independiente realizaron una evaluación de la calidad de los artículos seleccionados aplicando las guías de evaluación crítica de la JAMA. En los casos donde existieron discrepancias entre los evaluadores, estas fueron resueltas a través de consenso.

\section{RESULTADOS}

La búsqueda arrojó 1024 artículos de los cuales 34 cumplieron los criterios de inclusión,siendo finalmente 10 los artículos incluidos como evidencia (Fig. 1).

Veinticuatro artículos fueron excluidos posterior al análisis crítico por no responder la pregunta de in- 
vestigación, no cumplir los criterios de inclusión determinados para cada pregunta o por ser de baja calidad metodológica, esto es, ser sólo descriptivos o no establecer asociación entre variables, o por no presentar ajuste por variables de control.

Se identificaron 3 revisiones sistemáticas, de las cuales una, la del Gobierno Australiano (National Health and Medical Research Council, 2007), incluyó todos los efectos adversos estudiados. Cabe destacar también que la revisión del Gobierno Australiano, tomó como base la revisión de McDonagh et al., por ser considerada la revisión más completa realizada sobre los efectosde la fluoración del agua. Por lo tanto, la búsqueda de estudios originales realizados por ésta revisión incluyó estudios para el período 20002007, identificando sólo estudios no incluidos en la revisión de McDonagh et al. Ambas revisiones incluyeron estudios de todos los niveles de evidencia, inclusive estudios transversales y ecológicos, esto con la finalidad de permitir la más amplia búsqueda de evidencia.

Fluorosis dental. Siete estudios fueron incluidos como evidencia, dos revisiones sistemáticas (Khan et al.,
2005; National Health and Medical Research Council) $\mathrm{y}$ cinco estudios de corte transversal (Bottenberg et al., 2004; Cochran et al., 2004; Macpherson et al., 2007; Meyer-Lueckel et al., 2007; Whelton et al., 2006) (Tabla I), de los cuales se concluye que la fluoración del agua potable, a concentraciones de 0,6 a $1 \mathrm{ppm}$, se asocia a un mayor riesgo de fluorosis que la no fluoración. Sin embargo la mayoría de la fluorosis dental es del tipo cuestionable a leve y no es considerada un problema estético.

Es importante destacar que tanto en la revisión sistemática del Gobierno Australiano (National Health and Medical Research Council) como en la revisión publicada por Khan et al. (2005) los estudios primarios incluidos son de tipo descriptivos y que muy pocos de los estudios incluidos midieron el impacto de otras fuentes de incorporación de fluoruros o de otros posibles factores confundentes en la asociación entre fluorosis dental y fluoración del agua de beber a concentraciones de 0,6 a 1 ppm.

A pesar de que la mayoría de los estudios demostraron una mayor prevalencia de fluorosis en zonas fluoradas que no fluoradas, ésta fue principalmente

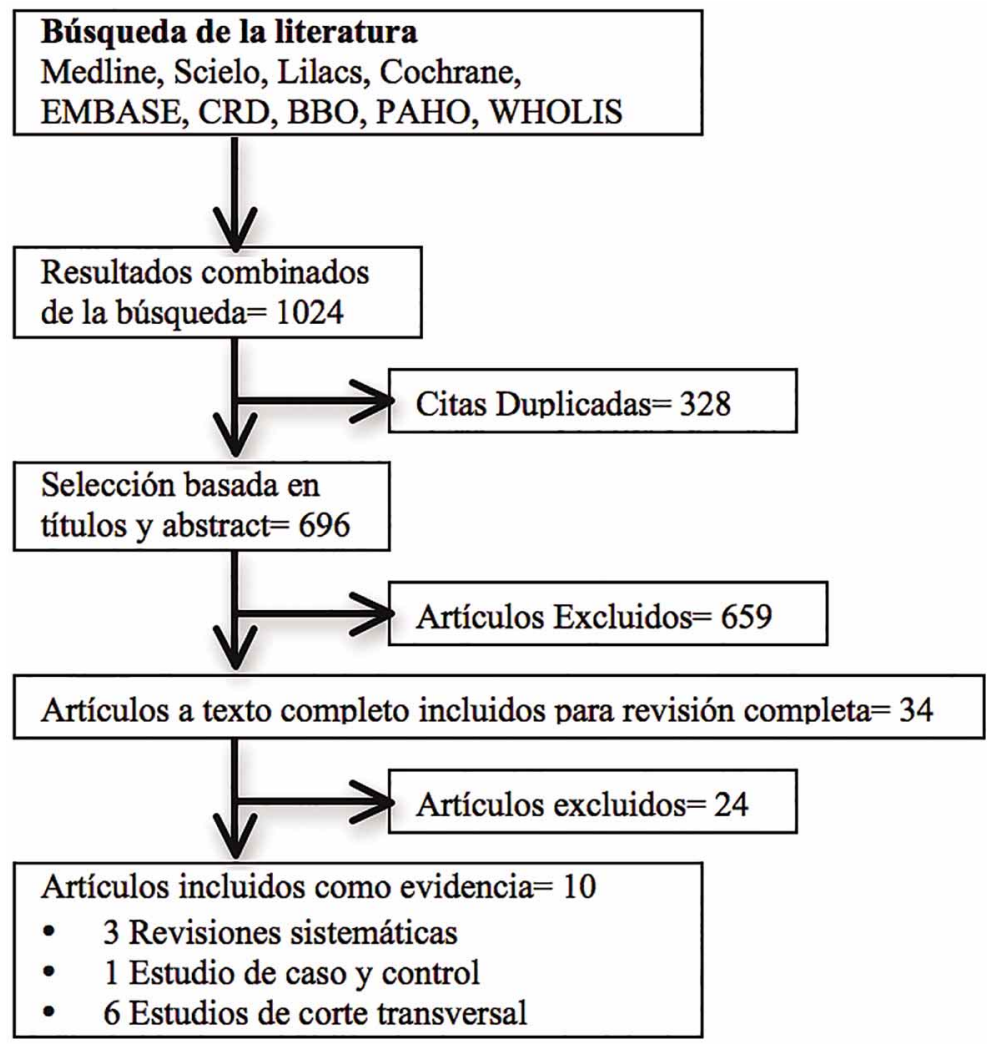

Fig. 1. Flujograma de búsqueda y selección. 


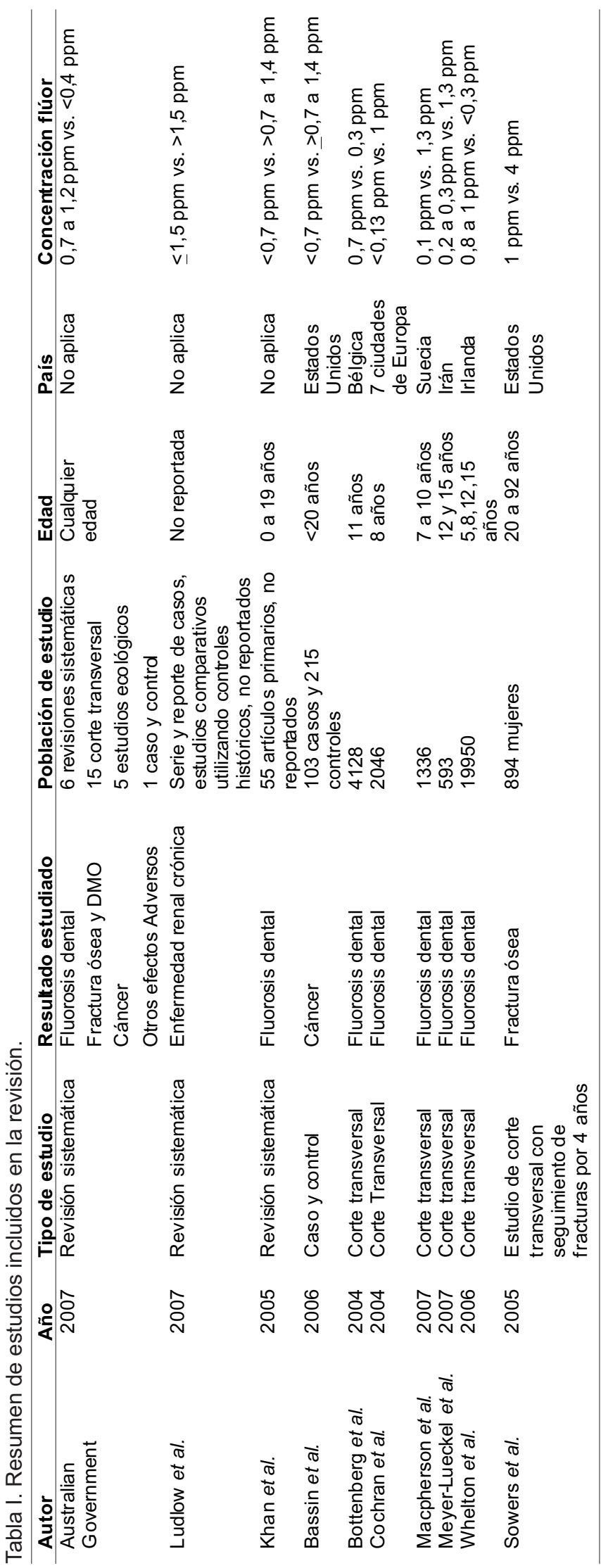

del tipo cuestionable a leve y la proporción de fluorosis con daño estético no difiere significativamente de la presente en zonas sin fluoración del agua. Según Macpherson et al. el incremento en la prevalencia de fluorosis, no se traduce en un significativo aumento en la percepción de una mayor necesidad de tratamiento.

Fracturas óseas. Una revisión sistemática ( National Health and Medical Research Council) y un estudio de corte transversal (Sowers et al., 2005) fueron incluidos como evidencia para establecer el efecto de la fluoración del agua de beber sobre de la densidad mineral ósea (DMO) y la fractura ósea (Tabla I). Si los estudios incluidos medían como resultado tanto la $\mathrm{DMO}$, como los resultados de fractura ósea, se informó ésta última de forma preferente, ya que esto representa el resultado más relevante del paciente.

De la revisión sistemática del Gobierno Australiano se concluye que la fluoración del agua potable, a concentraciones de 0,6 a $1 \mathrm{ppm}$, tiene poco efecto sobre el riesgo de fracturas ósea - ya sea protector o perjudicial en relación a la población sin fluoración delagua. Los estudios primarios incluidos en la revisión son estudios de corte transversal y no alteran los resultados de las revisiones sistemáticas incluidas.

El estudio de Sower et al., es un estudio predominantemente de corte transversal con seguimiento de las fracturas óseas por 4 años, las cuales fueron reportadas mediante vía correo cada 6 meses y confirmadas en el servicio médico donde fue tratada. Sus resultados muestran asociación significativa entre fracturas óseas y fluoración del agua pero por sobre los niveles óptimos (4 ppm). Sin embargo, esta diferencia no fue significativa al ajustar por covariables.

Cáncer. Para establecer la relación entre fluoración del agua y cáncer se incluyó una revisión sistemática (National Health and Medical Research Council) y un estudio de caso y control (Bassin et al., 2006) (Tabla I).

Los estudios incluidos no son concluyente en establecer asociación entre fluoración del agua, a concentraciones de 0,6 a $1 \mathrm{ppm}$ e incidencia global de cáncer al compararlo con la no fluoración del agua. Si bien el estudio de Bassin et al. muestra una asociación significativa entre exposición a fluoruros en el agua de beber e incidencia de osteosarcoma con un peak entre los 6-8 años en hombres diagnostica- 
dos antes de los 20 años de edad, la baja calidad metodológica del estudio nos hace mirar estos resultados con cautela. Los autores no encontraron ningún tipo de asociación en mujeres.

Otros Efectos Adversos. De las dos revisiones sistemáticas (National Health and Medical Research Council; Ludlow et al., 2007) incluidas para determinar la asociación entre fluoración del agua y cualquier otro efecto adverso se concluye que no hay evidencia de que el consumo de agua potable fluorada a concentraciones de 0,6 a $1 \mathrm{ppm}$, aumente el riesgo de desarrollar cualquier otro efecto adverso en la población general que la no fluoración del agua (Tabla I).

Las dos revisiones sistemáticas incluidas exploraron una serie de efectos adversos como síndrome de Down, Alzheimer, muerte fetal, disminución coeficiente intelectual, anomalías congénitas, enfermedad coronaria, retardo mental, fluorosis esqueletal, enfermedad renal crónica, entre otros, sin encontrar asociación significativa con concentraciones óptimas de ión fluoruro en el agua de beber.

\section{DISCUSIÓN}

La evidencia es clara en confirmar que la fluorosis dental es el efecto adverso más reportado asociado a la fluoración de agua, siendo la fluorosis un indicador de ingestión total de flúor durante el desarrollo dentario (McDonagh et al.; National Health and Medical Research Council; Parnell et al., 2009).

Tanto la revisión del Gobierno Australiano como la de Khan muestran una relación dosis-respuesta entre fluorosis y diferentes concentraciones de flúor en el agua, sin embargo, la severidad de fluorosis a concentraciones óptimas es principalmente de tipo muy leve a leve (McDonagh et al.; Khan et al., 2005; Whelton et al.). Si bien hay 4 veces más riesgo de desarrollar fluorosis con problemas estéticos en zonas de fluoración óptima versus subóptimas, el incremento absoluto en la prevalencia es de sólo un 4 a 5\% (National Health and Medical Research Council; Parnell et al.).

Es importante resaltar que en ambas revisiones sistemáticas, los estudios primarios incluidos fallan en el esfuerzo de controlar los efectos de potenciales factores confundentes, lo que no descarta que este aumento de la prevalencia se deba a otras fuen- tes de exposición al flúor y no sólo a la fluoración del agua. Esto es reforzado por la evidencia que muestra un aumento de fluorosis tanto en zonas fluoradas como no fluoradas, aunque no estadísticamente significativo (Khan et al., 2005; Mascarenhas \& Burt).

Los resultados de esta revisión concuerdan con datos aportados por las revisiones de Jones et al. (1999) y de Demos et al. (2001) sobre asociación entre fluoración del agua y fracturas óseas. Ellos concluyen que la fluoración del agua a niveles para prevenir caries no tiene un efecto adverso sobre la DMO o la incidencia de fracturas. Demos et al. encontró sólo un estudio que mostró un incremento en la incidencia de fracturas pero a niveles 4 veces mayor a los niveles considerados como óptimos. Al igual que el estudio de Sowers et al. (2005), el control de la variables confundentes fue pobre, lo que nos indica que esta asociación se pude deber a otro factor confundente y no sólo a la concentración del flúor en el agua.

La evidencia tampoco es concluyente en establecer que la fluoración del agua puede tener un efecto protector en la incidencia de fracturas óseas (McDonagh et al.; National Health and Medical Research Council; Jones et al.; Demos et al.).

Numerosos estudios han fallado en demostrar la asociación entre los diferentes niveles de flúor en el agua e incidencia global de cáncer (McDonagh et al.; National Health and Medical Research Council; Gelberg et al., 1995; Kim et al., 2011). En nuestra revisión sólo el estudio de Bassin et al. muestra un incremento del riesgo entre un subconjunto de hombres expuestos al flúor en el agua potable durante la infancia y osteosarcoma. Este estudio presenta sólo hallazgos parciales del proyecto de investigación sobre fluoruros y osteosarcoma realizado por la escuela de Medicina Oral de la Universidad de Harvard. Es por esta razón, que varios autores (Kim et al.; Douglass \& Joshipura, 2006) invitan a mirar estos resultados con cautela.

Douglass \& Joshipura, argumentan que los resultados preliminares del análisis global de los casos identificados entre 1993 y 2000 (segundo grupo de casos) no muestran una asociación entre el osteosarcoma y flúor en el agua potable. Además expone que los casos fueron identificados a partir de los mismos hospitales dentro de los mismos departamentos de ortopedia y que los mismos departamentos de patología hacen el diagnóstico de osteosarcoma, lo que podría afectar los resultados del estudio. 
Además argumenta que estudios preliminares de las muestras óseas que se proporcionan para muchos de los casos, sugieren que el nivel de fluoruro en el hueso no está asociado con exceso de riesgo de osteosarcoma. Esto es confirmado posteriormente por Kim et al.

Bassin et al., dentro de sus limitaciones expone que las estimaciones de fluoruro en el agua potable en cada residencia no reflejan el consumo real de flúor, que hay una falta de datos sobre otros posibles factores de confusión, tales como otras fuentes de ingestión de fluoruros o la presencia de algún tipo agentes contaminante o cancerígenos presente en el agua o alimentos.

Con respecto a otros posibles efectos adversos, la evidencia es demasiado limitada para permitir conclusiones seguras. Esta revisión confirma los hallazgos de revisiones previas sobre el tema y por tal razón no existe evidencia que sugiera el estudio de algún otro efecto adverso de forma prioritaria.

ZAROR, C.; VALLEJOS, C.; CORSINI, G.; DE LA PUENTE, C.; VELÁSQUEZ, M.;TESSADA-SEPÚLVEDA, R.; DEL VALLE-ARANDA, C.;VEGA-RODRÍGUEZ, G. Systematic review of the adverse effects of water fluoridation. Int. J. Odontostomat., 9(1):165-171, 2015.

ABSTRACT: During the last decades, considerable scientific attention has been paid to the safety of fluoride, given the wide variety of sources of intake at which the population is exposed and the risks to the health of people this may produce. The aim was to determine whether water fluoridation at concentrations from 0.6 to $1 \mathrm{ppm}$ is associated with a higher proportion of adverse effects in the general population when comparing them to suboptimal concentrations. A systematic review was conducted of the literature in MEDLINE, EMBASE, COCHRANE, SCIELO, LILACS, CRD, BBO, PAHO and WHOLIS, limited to 2002 to 2012. Included were primary and secondary studies in Spanish, English and Portuguese with at least two compared populations, one with optimal fluoride levels in the water $(0.6-1 \mathrm{ppm})$ and another without water fluoridation $(<0.3 \mathrm{ppm})$ or with suboptimal levels $(>0.3<0.6 \mathrm{ppm})$. Two researchers independently evaluated the quality of the articles selected and which met the inclusion criteria. The search revealed 1024 articles, of which 24 met the inclusion criteria and 10 were included as evidence. With the exception of dental fluorosis, there is no association between any other adverse effect and water fluoridation. Despite the greater prevalence of fluorosis in fluoride than in non-fluoride zones, this was mainly questionable to slight and the proportion of fluorosis with esthetic damage does not differ significantly from this in zones without water fluoridation

KEY WORDS: fluoride, drinking water, fluorosis, adverse effects.

\section{REFERENCIAS BIBLIOGRÁFICAS}

Armfield, J. M. Community effectiveness of public water fluoridation in reducing children's dental disease. Public Health Rep., 125(5):655-64, 2010.

Bassin, E. B.; Wypij, D.; Davis, R. B. \& Mittleman, M. A. Agespecific fluoride exposure in drinking water and osteosarcoma (United States). Cancer Causes Control, 17(4):421-8, 2006.

Bottenberg, P.; Declerck, D.; Ghidey, W.; Bogaerts, K.; Vanobbergen, J. \& Martens, L. Prevalence and determinants of enamel fluorosis in Flemish schoolchildren. Caries Res., 38(1):20-8, 2004.

Centers for Disease Control and Prevention (CDC). Ten great public health achievements--United States, 1900-1999. M. M. W. R. Morb. Mortal Wkly. Rep., 48(12):241-3, 1999.

Clark, D. C.; Shulman, J. D.; Maupomé, G. \& Levy, S. M. Changes in dental fluorosis following the cessation of water fluoridation. Community Dent. Oral Epidemiol., 34(3):197-204, 2006.
Cochran, J. A.; Ketley, C. E.; Arnadóttir, I. B.; Fernades, B.; Koletsi-Kounari, H.; Oila, A.; van Loveren, C.; Whelton, H. P. \& O'Mullane, D. M. A comparison of the prevalence of fluorosis in 8-year-old children from seven European study sites using a standardized methodology. Community Dent. Oral Epidemiol., 32 (Suppl. 1):28-33, 2004.

Demos, L. L.; Kazda, H.; Cicuttini, F. M.; Sinclair, M. I. \& Fairley, C. K. Water fluoridation, osteoporosis, fractures-recent developments. Aust. Dent. J., 46(2):80-7, 2001.

Denbesten, P. \& Li, W. Chronic fluoride toxicity: dental fluorosis. Monogr. Oral Sci., 22:81-96, 2011.

Douglass, C. W. \& Joshipura, K. Caution needed in fluoride and osteosarcoma study. Cancer Causes Control, 17(4):481-2, 2006.

Gelberg, K. H.; Fitzgerald, E. F.; Hwang, S. A. \& Dubrow, R. Fluoride exposure and childhood osteosarcoma: a casecontrol study. Am. J. Public Health., 85:1678-83, 1995. 
Jones, G.; Riley, M.; Couper, D. \& Dwyer, T. Water fluoridation, bone mass and fracture: a quantitative overview of the literature. Aust. N. Z. J. Public Health, 23(1):34-40, 1999.

Khan, A. A.; Whelton, H. \& O'Mullane, D. Determining the optimal concentration of fluoride in drinking water in Pakistan. Community Dent. Oral Epidemiol., 32(3):16672, 2004.

Khan, A.; Moola, M. H. \& Cleaton-Jones, P. Global trends in dental fluorosis from 1980 to 2000: a systematic review. S. A. D. J., 60(10):418-21, 2005.

Kim, F. M.; Hayes, C.; Williams, P. L.; Whitford, G. M.; Joshipura, K. J.; Hoover, R. N.; Douglass, C. W. \& National Osteosarcoma Etiology Group. An assessment of bone fluoride and osteosarcoma. J. Dent. Res., 90(10):1171-6, 2011.

Levy, S. M.; Warren, J. J.; Davis, C. S.; Kirchner, H. L.; Kanellis, M. J. \& Wefel, J. S. Patterns of fluoride intake from birth to 36 months. J. Public Health Dent., 61(2):707, 2001.

Levy, S. M.; Kohout, F. J.; Guha-Chowdhury, N.; Kiritsy, M. C.; Heilman, J. R. \& Wefel, J. S. Infants' fluoride intake from drinking water alone, and from water added to formula, beverages, and food. J. Dent. Res., 74(7):1399407, 1995.

Ludlow, M.; Luxton, G. \& Mathew, T. Effects of fluoridation of community water supplies for people with chronic kidney disease. Nephrol. Dial. Transplant., 22(10):2763-7, 2007.

Macpherson, L. M.; Conway, D. I.; Gilmour, W. H.; Petersson, L. G. \& Stephen, K. W. Photographic assessment of fluorosis in children from naturally fluoridated Kungsbacka and non-fluoridated Halmstad, Sweden. Acta Odontol. Scand., 65(3):149-55, 2007.

Mascarenhas, A. K. \& Burt, B. A. Fluorosis risk from early exposure to fluoride toothpaste. Community Dent. Oral Epidemiol., 26(4):241-8, 1998.

McDonagh, M. S.; Whiting, P. F.; Wilson, P. M.; Sutton, A. J.; Chestnutt, I.; Cooper, J.; Misso, K.; Bradley, M.; Treasure, E. \& Kleijnen, J. Systematic review of water fluoridation. B. M. J., 321(7265):855-9, 2000.

Meyer-Lueckel, H.; Bitter, K.; Shirkani, B.; Hopfenmuller, W. \& Kielbassa, A. M. Prevalence of caries and fluorosis in adolescents in Iran. Quintessence Int., 38(6):459-65, 2007.

National Health and Medical Research Council. A Systematic Review of the Efficacy and Safety of Fluoridation. Part A: Review of Methodology and Results. Canberra, Australian Government, 2007. Disponible en: http:// www.nhmrc.gov.au/files_nhmrc/publications/ attachments/eh41_1.pdf

Parnell, C.; Whelton, H. \& O'Mullane, D. Water fluoridation. Eur. Arch. Paediatr. Dent., 10(3):141-8, 2009.

Sowers, M.; Whitford, G. M.; Clark, M. K. \& Jannausch, M. $\mathrm{L}$. Elevated serum fluoride concentrations in women are not related to fractures and bone mineral density. J. Nutr., 135(9):2247-52, 2005

Vallejos, V. C.; Lorca, O. P. \& Orellana. C. J. J. Análisis de la evidencia publicada sobre efectividad y riesgos de la fluoración del agua potable para la prevención de caries dentales. Santiago de Chile, Ministerio de Salud de Chile, Universidad de La Frontera, 2002. Disponible en http:/ / w e b. m in s a I. cl/ portal/ u rl/ i t e m / a1eb519e7e9c4592e04001011 f01798b.pdf

Whelton, H.; Crowley, E.; O'Mullane, D.; Donaldson, M.; Cronin, M. \& Kelleher, V. Dental caries and enamel fluorosis among the fluoridated population in the Republic of Ireland and non fluoridated population in Northern Ireland in 2002. Community Dent. Health., 23(1):37-43, 2006.

Dirección para Correspondencia:

Prof. Carlos Zaror Sánchez

Facultad de Odontología

Universidad de la Frontera

Manuel Montt \#112

Temuco

CHILE

Email: carlos.zaror@ufrontera.cl

Recibido : 19-12-2014

Aceptado: 26-03-2015 